\title{
The COL5A1 gene and musculoskeletal soft-tissue injuries
}

\author{
Michael Posthumus (PhD) $)^{1,3}$ \\ Alison V September (PhD) ${ }^{1,3}$ \\ Martin P Schwellnus (MB BCh, MSc (Med), MD, FACSM, FFIMS) $)^{1,3}$ \\ Malcolm Collins (PhD) ${ }^{2,1,3}$ \\ ${ }^{1}$ UCT/MRC Research Unit for Exercise Science and Sports Medicine, Department of Human Biology, Faculty of Health Sciences, University of \\ Cape Town \\ ${ }^{2}$ South African Medical Research Council, Cape Town \\ ${ }^{3}$ The International Olympic Committee Centre of Excellence, Newlands, Cape Town
}

\begin{abstract}
Background. It has been shown that there is an association between various genetic variants and Achilles tendon injuries as well as anterior cruciate ligament (ACL) ruptures. Among other variants the BstUI restriction fragment length polymorphism (RFLP) within the COL5A1 gene has been shown to be over-represented in asymptomatic participants when compared with those with chronic Achilles tendinopathy, and in asymptomatic female participants when compared with those with $\mathrm{ACL}$ ruptures. The male asymptomatic control participants in the ACL study, which were 10 years younger than previously investigated cohorts, had a distinctly different genotype frequency.

Aim. The aim of this study was therefore to determine whether the distribution of the COL5A1 BstUI RFLP in the combined asymptomatic participants without any known history of tendon injuries is age dependent, particularly among males.

Results. When the 265 male asymptomatic participants from all studies were pooled and divided into age-group tertiles, there was a significant linear increase in the CC genotype frequency $(p=0.032)$ among the male age groups, with the youngest group having the lowest frequency (CC genotype frequency, 13\%) and the oldest group having the highest (CC genotype frequency, $27 \%$ ) frequency. There was however a similar CC genotype content in all three female $(N=231)$ age groups (CC genotype frequency, $24-27 \%$; $p=0.795$ ).
\end{abstract}

\section{CORRESPONDENCE:}

Dr Michael Posthumus

UCT/MRC Research Unit for Exercise Science and Sports

Medicine

PO Box 115

Newlands, 7725

Tel: +27 216504572

Fax: +27216867530

E-mail: mposthumus@mweb.co.za
Conclusion. The practical implication is that the selection of asymptomatic groups is of critical importance when future studies of this nature are designed. Future research investigating this genetic variant as a risk factor for soft-tissue injuries should consider these findings when selecting asymptomatic participants.

\section{Introduction}

Acute and chronic musculoskeletal soft-tissue injuries are common during participation in physical activity. ${ }^{1}$ Multiple extrinsic and intrinsic risk factors are implicated in the aetiology of these complex injuries. $^{2,3}$ In two specific injuries, anterior cruciate ligament (ACL) ruptures and chronic Achilles tendinopathy, genetic components have been identified as intrinsic risk factors. Among the genetic risk factors identified, the CO5A1 BstUI restriction fragment length polymorphism (RFLP) has been associated with both chronic Achilles tendinopathy and ACL ruptures. ${ }^{3-5}$

The CC genotype of the COL5A1 BstUI RFLP was significantly over-represented in asymptomatic participants compared with those with chronic Achilles tendinopathy - both in South African ${ }^{4}$ and Australian ${ }^{3}$ populations. A similar finding was reported when female participants with $A C L$ ruptures were compared with asymptomatic female controls. ${ }^{5}$ All control groups in these studies were matched for physical activity and physiological characteristics. These data suggest that individuals with a CC genotype are protected, despite the particular load and/or external forces applied to their musculoskeletal soft tissues. However, the CC genotype of the COL5A1 BstUI RFLP was not over-represented in male subjects with $\mathrm{ACL}$ ruptures compared with asymptomatic male controls. ${ }^{5}$ Owing to the reported increased risk of $A C L$ ruptures among females, the $\mathrm{ACL}$ study analysed males and females separately. The previous two Achilles tendinopathy studies only analysed males and females as one group.

Interestingly, the CC genotype frequency of the male asymptomatic participants of the ACL study was distinctly lower than the CC genotype frequencies of the asymptomatic control cohorts in which the CC genotype was over-represented. ${ }^{3-5}$ Furthermore, the male asymptomatic participants of the ACL study were approximately 10 years younger than the asymptomatic control groups in the previous two Achilles tendinopathy studies. 


\begin{tabular}{|c|c|c|c|c|}
\hline & $\leq 25$-year group & 26 - 42-year group & $\geq 43$-year group & $p$-value \\
\hline Males & $N=83$ & $N=112$ & $N=70$ & \\
\hline \multirow[t]{2}{*}{ Age (yrs) } & $22.3 \pm 1.7^{* \dagger}$ & $33.0 \pm 5.0^{\star *}$ & $52.6 \pm 8.2^{\dagger \ddagger}$ & \\
\hline & $(18-25)$ & $(26-42)$ & $(43-77)$ & $<0.001$ \\
\hline \multirow[t]{2}{*}{ Height (cm) } & $180 \pm 6$ & $180 \pm 6$ & $178 \pm 7$ & \\
\hline & $(168-195)$ & $(167-201)$ & $(164-195)$ & 0.590 \\
\hline \multirow[t]{2}{*}{ Weight (kg) } & $79.9 \pm 11.5$ & $81.4 \pm 12.2$ & $81.9 \pm 15.0$ & \\
\hline & $(61.7-110.0)$ & $(59.7-137.0)$ & $(58.0-136.0)$ & 0.065 \\
\hline \multirow[t]{2}{*}{$\mathrm{BMI}\left(\mathrm{kg} / \mathrm{m}^{2}\right)$} & $24.5 \pm 2.8^{\S}$ & $25.2 \pm 3.4$ & $26.0 \pm 4.3^{\S}$ & \\
\hline & $(18.5-31.7)$ & $(19.9-38.4)$ & $(20.2-39.3)$ & 0.036 \\
\hline South African born (\%) & $79.5^{\star \dagger}$ & $47.7^{\star}$ & $40.5^{\dagger}$ & $<0.001$ \\
\hline Australian born (\%) & 10.3 & 29.4 & 36.2 & \\
\hline Females & $\mathrm{N}=63$ & $N=108$ & $N=60$ & \\
\hline \multirow[t]{2}{*}{ Age (yrs) } & $22.6 \pm 1.7^{\star \dagger}$ & $32.4 \pm 4.5^{\star \pm}$ & $52.0 \pm 6.7^{\dagger \ddagger}$ & \\
\hline & $(19-25)$ & $(26-42)$ & $(43-72)$ & $<0.001$ \\
\hline \multirow[t]{2}{*}{ Height (cm) } & $166 \pm 7$ & $168 \pm 7^{\ddagger}$ & $164 \pm 8^{\ddagger}$ & \\
\hline & $(152-179)$ & $(152-187)$ & $(145-181)$ & 0.004 \\
\hline \multirow[t]{2}{*}{ Weight (kg) } & $61.2 \pm 6.5$ & $64.4 \pm 9.0$ & $81.9 \pm 15.0$ & \\
\hline & $(49.0-79.2)$ & $(48.0-87.0)$ & $(47.0-115.0)$ & 0.051 \\
\hline \multirow[t]{2}{*}{ BMI $\left(\mathrm{kg} / \mathrm{m}^{2}\right)$} & $22.1 \pm 2.1^{\dagger}$ & $22.9 \pm 3.0$ & $24.1 \pm 4.6^{\dagger}$ & \\
\hline & $(18.1-28.1)$ & $(18.1-33.2)$ & $(18.6-46.7)$ & 0.005 \\
\hline South African born (\%) & $66.1^{* \dagger}$ & $31.5^{*}$ & $21.7^{\dagger}$ & $<0.001$ \\
\hline Australian born (\%) & 25.4 & 48.2 & 56.7 & \\
\hline
\end{tabular}

The objective of this study was therefore to further examine the age- and sex-related changes in the COL5A1 BstUI RFLP genotype frequency among the combined asymptomatic participants. More specifically, our primary aim was to determine if the CC genotype frequency of the COL5A1 BstUI RFLP among male and female subjects without a previous history of tendon injury is age dependent.

\section{Methods}

All 496 asymptomatic participants (265 male and 231 female) without a reported history of tendon injuries that were previously investigated in three separate publications were included in this analysis. ${ }^{3-5}$ Because of the design of the previous tendinopathy studies, it was not possible to exclude those with a history of ligament injuries. Prior to participation in these original studies, all participants gave written informed consent and completed a medical history questionnaire form. All descriptive data for the subjects with Achilles tendinopathy, as well as the asymptomatic control groups, were previously reported. ${ }^{3-5}$ All three studies were approved by the Research Ethics Committee of the Faculty of Health Sciences of the University of Cape Town and/or the Human Ethics Committee of La Trobe University, Melbourne, Australia.
The participants were combined and divided into three male and three female age groups: (i) $\leq 25$ years old; (ii) 26 - 42 years old; and (iii) $\geq 43$ years old. A chi-squared $\left(\chi^{2}\right)$ test, Fisher's exact test or $\chi^{2}$ test for linear trends was used to analyse differences in genotype and any other categorical data between the groups. Data were analysed using STATISTICA Version 8.0 (Statsoft Inc., Tulsa, Oklahoma, USA) and Graphpad InStat Version 3 (Graphpad Software, San Diego, California, USA) statistical programs. Statistical significance was accepted when $p<0.05$. A one-way analysis of variance (ANOVA) was used to determine any significant difference between the characteristics of the male and female age groups. Hardy-Weinberg equilibrium values were established using the program Genepop web version 3.4 (http://genepop.curtin.edu.au/).

\section{Results}

There was a significant linear trend $(p=0.032)$ for the CC genotype frequency among the male age groups (Fig. 1A). The youngest group had the lowest CC frequency (13\%), and the oldest group the highest CC frequency (27\%) (Fig. 1A). The CC genotype content in all three female age groups $(24-27 \%)$ was similar $(p=0.795$, Fig. 1B). The general characteristics of the male and female age group tertiles are described in Table I. 


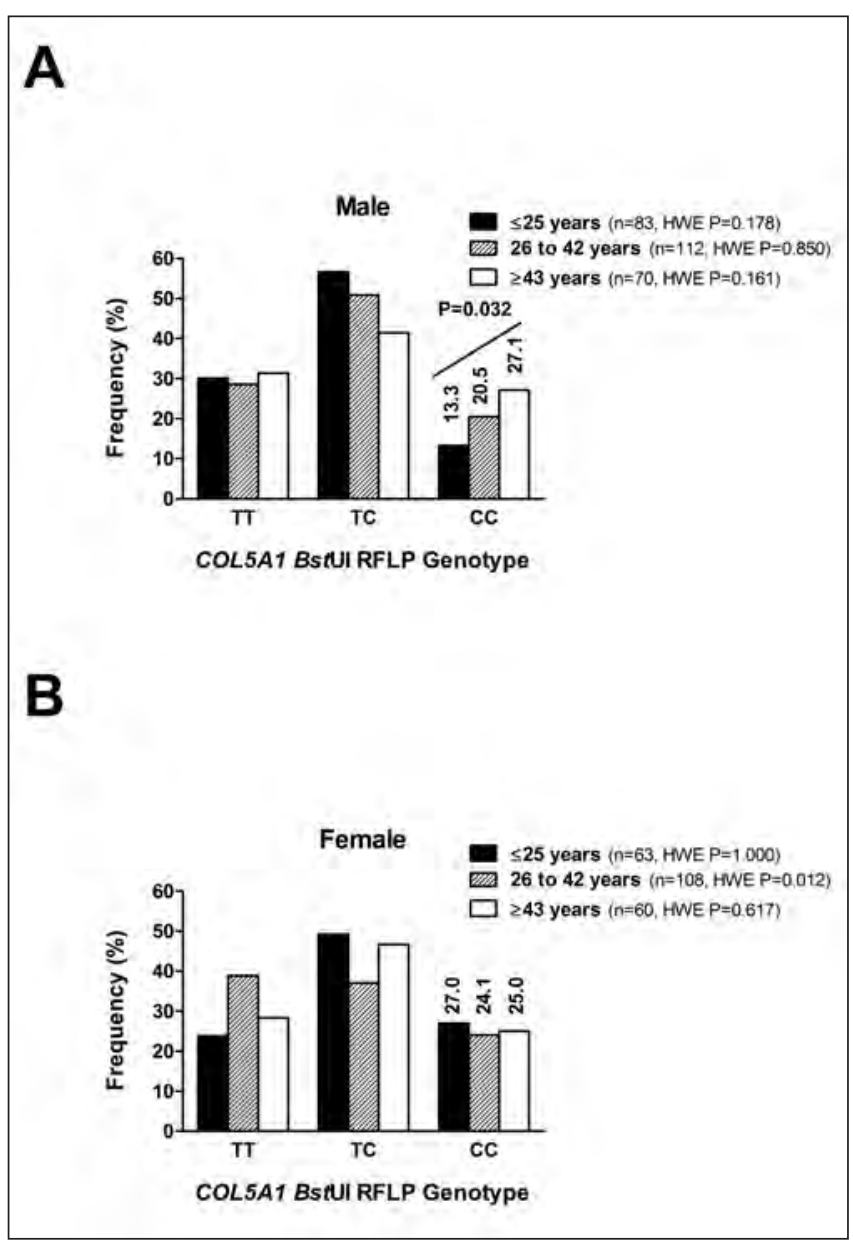

Fig. 1. The genotype frequency of the COL5A1 BstUI restriction fragment length polymorphism (RFLP) in all (A) male and (B) female asymptomatic participants divided according to age into participants ( $\leq 25$ years old black bars), 26 - 42 years old (thatched bars), and $>42$ years old (clear bars). A significant linear trend $(p=0.032)$ for the CC genotype content among the male age groups was found. The number of subjects $(\mathrm{N})$ within each category, as well as the Hardy-Weinberg equilibrium (HWE) p-values, are shown in parentheses.

\section{Discussion}

The main finding of this study was that there is a significant agedependent increase in the distribution of the COL5A1 BstUI RFLP CC genotype in the pooled asymptomatic male participants of the three studies which investigated this polymorphism as a possible risk factor for musculoskeletal soft-tissue injuries. No similar trends were observed in the female subjects.

We propose that the reported finding indicates that the youngest group of asymptomatic male participants consists of a mixture of individuals, similar to the general population, who are at low and high risk of musculoskeletal soft-tissue injuries (Fig. 2). However, when older subjects (who would have had a greater amount of exposure to extrinsic factors) are analysed, individuals who may have been previously uninjured, would have developed an injury. Therefore, when older asymptomatic participants are analysed, the group will contain a highly selected sample of the population at low risk of musculoskeletal soft-tissue injuries. This could explain the finding of a significant linear trend in the COL5A1 BstUI RFLP CC genotype

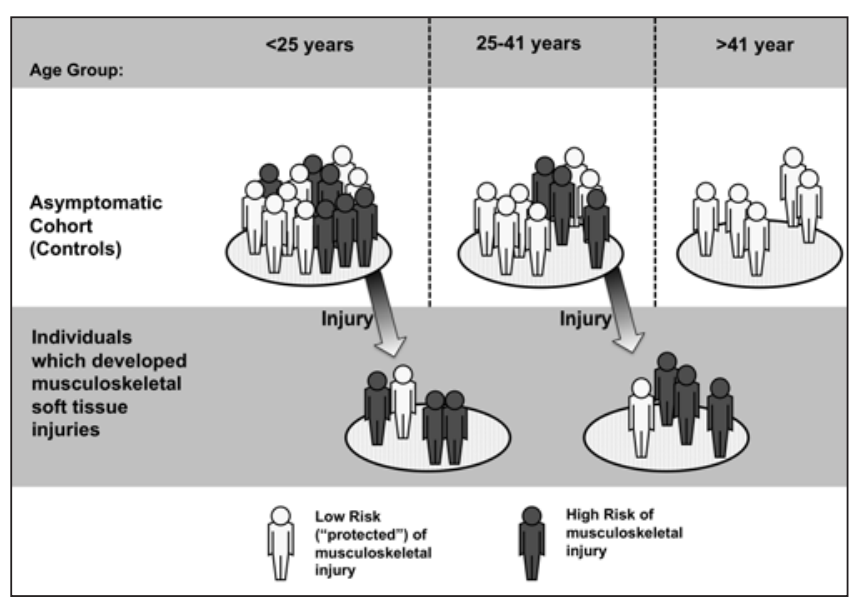

Fig. 2. Proposed explanation for the significant linear trend in CC genotype frequency among the asymptomatic male subjects when divided into the three age groups ( $<25$ years, 25 - 41 years, and $>41$ years). It is proposed that asymptomatic subjects in the age category $<25$ years will more than likely consist of individuals at high (black shaded) and low (no shade) risk of musculoskeletal soft-tissue injury. Among older asymptomatic groups of participants ( 25 - 41 years, and $>41$ years) the relative proportion of individuals at high risk of injury will be reduced, as the likelihood of high-risk individuals becoming injured over time is greater than the likelihood of low-risk individuals becoming injured.

frequency and an increased chronological age in the male subjects analysed. This proposed explanation is further supported by a departure from Hardy-Weinberg equilibrium as observed in some of the groups presented in our previous studies. ${ }^{3}$

It remains unknown why a similar trend was not observed in females. It does however suggest that the COL5A1 BstUI RFLP, as a risk factor for musculoskeletal soft-tissue injuries, is not age dependent in females.

A limitation of this study was that it was not possible to analyse the South African and Australian data separately owing to small sample sizes and uneven genotype distribution. A further limitation was that, although all participants were asymptomatic with regard to a previous history of tendon injuries, ${ }^{3-5}$ not all were free of ligament injuries (owing to the study designs).

In conclusion, there was an age-dependent significant increase in distribution of the COL5A1 BstUI RFLP CC genotype in the pooled asymptomatic male participants of the three studies which previously investigated this polymorphism as a possible risk factor for soft-tissue injuries. The practical implication of this finding is that the selection of control groups is of critical importance when future studies of this nature are designed. Future research investigating this genetic variant as a risk factor for soft-tissue injuries should consider the findings of this study when selecting an asymptomatic control group.

\section{Perspective}

Genetic variants, such as the COL5A1 BstUI RFLP, may have a significant impact on the prevention of musculoskeletal soft-tissue injures. ${ }^{6}$ Genetic variants, together with other intrinsic and extrinsic risk factors, should eventually be used to identify individuals at increased risk of injury. Once individuals are identified as 'at risk', carefully designed intervention programmes should be prescribed to 
prevent the injury from occurring and to assist the clinical management of these individuals. The current study provides further information on the COL5A1 BstUI RFLP. The findings may help future studies investigating this genetic variant as a risk factor for musculoskeletal soft-tissue injuries and thereby assist future multifactorial risk models.

\section{Acknowledgements}

This study was supported in part by funds from the National Research Foundation (NRF) of South Africa (Grant No. FA2005021700015 and FA2007032700010), the University of Cape Town, and the South African Medical Research Council (MRC). AVS was supported by the postdoctoral innovation award of the NRF.

Competing interests. None.

\section{REFERENCES}

1. Clayton RA, Court-Brown CM. The epidemiology of musculoskeletal tendinous and ligamentous injuries. Injury 2008;39(12):1338-1344.

2. Meeuwisse WH. Assesing causation in sport injury: a multifactorial model. Clin J Sport Med 1994;4:166-1670.

3. September AV, Cook J, Handley CJ, Van der Merwe L, Schwellnus MP, Collins M. Variants within the COL5A1 gene are associated with Achilles tendinopathy in two populations. Br J Sports Med 2009;43(5):357-365.

4. Mokone GG, Schwellnus MP, Noakes TD, Collins M. The COL5A1 gene and Achilles tendon pathology. Scand J Med Sci Sports 2006;16(1):1926.

5. Posthumus $M$, September AV, O'Cuinneagain $D$, van der Merwe $W$, Schwellnus MP, Collins M. The COL5A1 gene is associated with increased risk of anterior cruciate ligament ruptures in female participants. Am J Sports Med 2009;37(11):2234-2240.

6. Collins M. Genetic risk factors for soft tissue injuries 101: A practical summary to help clinicians understand the role of genetics and 'personalised medicine'. Br J Sports Med (in press) Available on EPUB: http://www. ncbi.nlm.nih.gov/pubmed/19553227

\section{The ESSENTIAL REFERENCE for every healthcare professional!}

The carefully and thoroughly updated $9^{\text {th }}$ edition of the South African Medicines Formulary (SAMF) can now be ordered. It is your essential reference to rational, safe and cost-efficient use of medicines. That is why you should not prescribe without it.

The newly published SAMF provides easy access to the latest, most scientifically accurate information - including full drug profiles, clinical notes and special prescriber's points. The convenient pocket-size design enables you to fit it comfortably into your bag or hospital coat pocket - always at hand for ready reference.

\section{WHYY YOU SHOULDNT BE WITHOUT THE SAMF 9 $^{\text {TH }}$ EDITION}

The new $9^{\text {th }}$ edition of SAMF provides expanded information on key issues facing South African healthcare professionals today, including antiretrovirals, TB treatment guidelines, management guidelines for asthma and chronic heart failure, other common chronic conditions and prescribing in sport.

- It presents practical, new approaches to the management of venomous bites and stings.

- It outlines extensively the acute adverse reactions to drugs of abuse, and their management.

- It features new as well as existing drugs, indexed by both trade and generic names.

- It offers fresh insights into informed prescribing and carries cautionary guidelines on drug interactions and a range of special risk patients and conditions.

And, as always, you can rely on...

- the professional compilation and editing by a team from the Division of Clinical Pharmacology, UCT

- $\quad$ an independent and unbiased guide on prescribing in South Africa today

- the indication of agents included in the SA and WHO essential drug lists

- $\quad$ support of the SA national drug policy

- $\quad$ guidance for prescribing during pregnancy and lactation, and in patients with porphyria, liver disease and renal impairment (including tables with drug dosage adjustments); and

- indexed and page tabs for quick and easy access to each section.

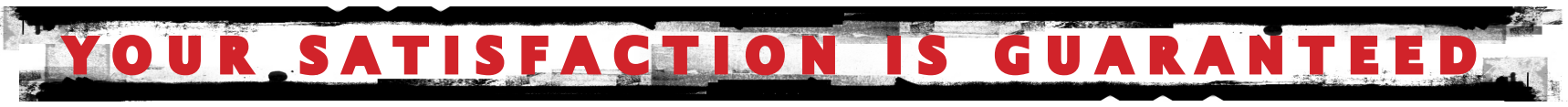

\section{3 easy order options:}

1. PHONE EDWARD OR BYRON - 0216817000

2. FAX the completed SAMF order form to 0866006218

3. EMAIL: edwardm@hmpg.co.za OR byronm@hmpg.co.za 